\title{
Light weight silicon mirrors for space instrumentation
}

Each mirror is a monolithic structure from a single crystal of silicon

The mirrors are light weighted after the optical surface is ground and polished

Mirrors made during the initial phase of this work were typically $1 / 50 \lambda$ or better (RMS at $633 \mathrm{~nm}$ )

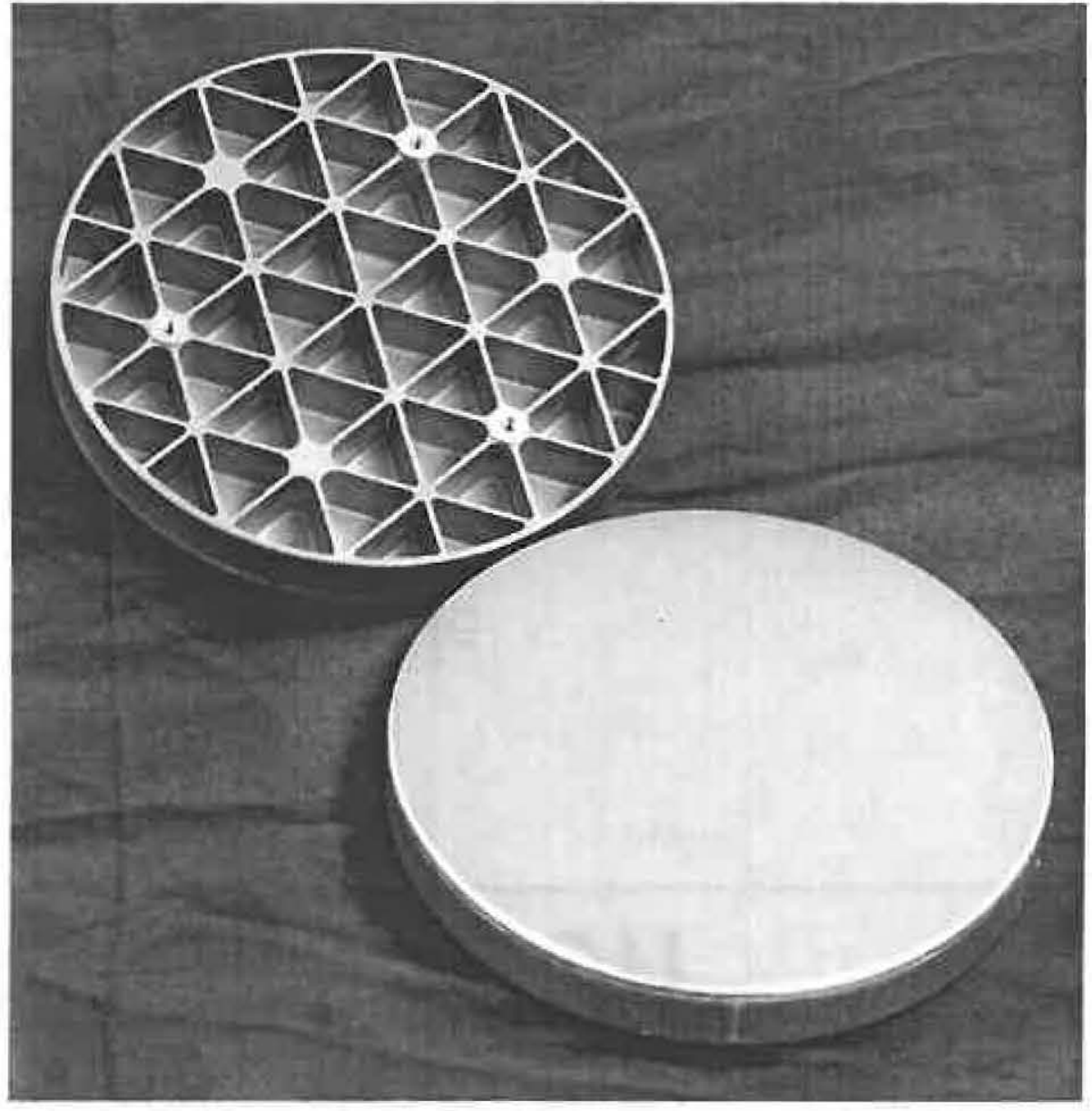




\section{Light weight silicon mirrors for space instrumentation}

Steps in Initial Process:

1: Form a blank from a single crystal silicon boule

2: Heat treat the blank at $1250 \mathrm{C}$ to heal crystalline damage caused by the sawing and grinding used to form the blank

3: Form the optical surface by conventional grinding \& polishing

4: Bond a protector disk to the optical surface using stacking wax

5: Light weight the mirror using ultrasonic maching to form an isogrid pattern

6: Remove the protector and heat treat again to heal crystalline damage caused by the light weighting process 


\section{Light weight silicon mirrors for space instrumentation}
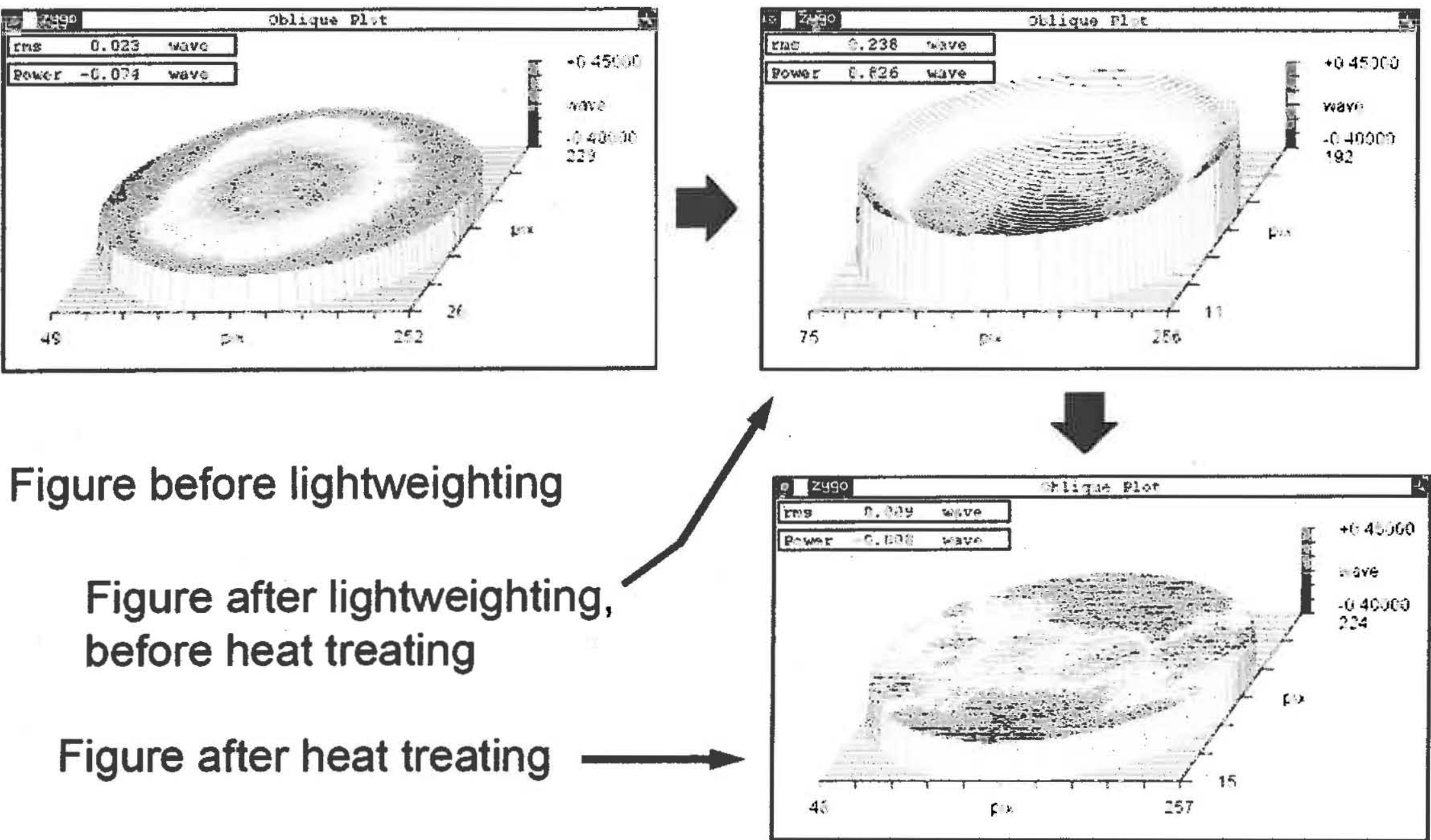

Figure before lightweighting

Figure after lightweighting, before heat treating

Figure after heat treating 


\section{Light weight silicon mirrors for space instrumentation}

CIRS-Lite Cassegrain Primary

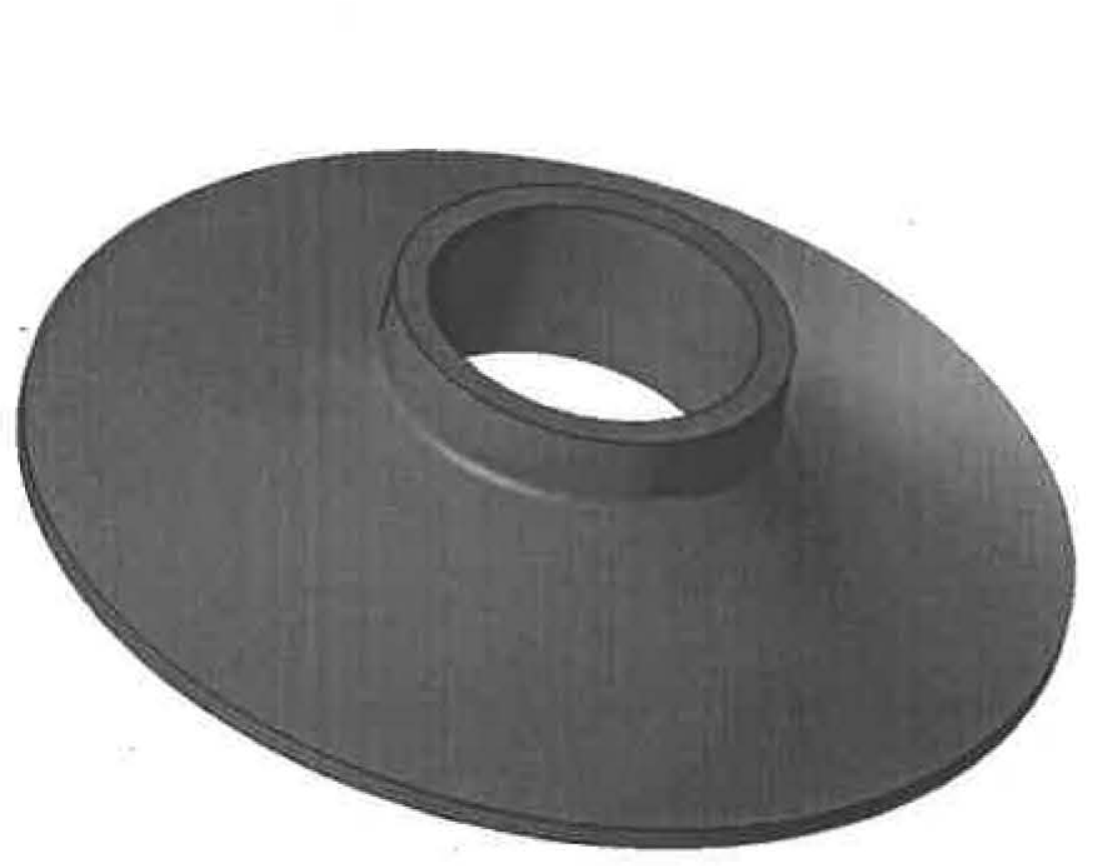

Rear View (model)

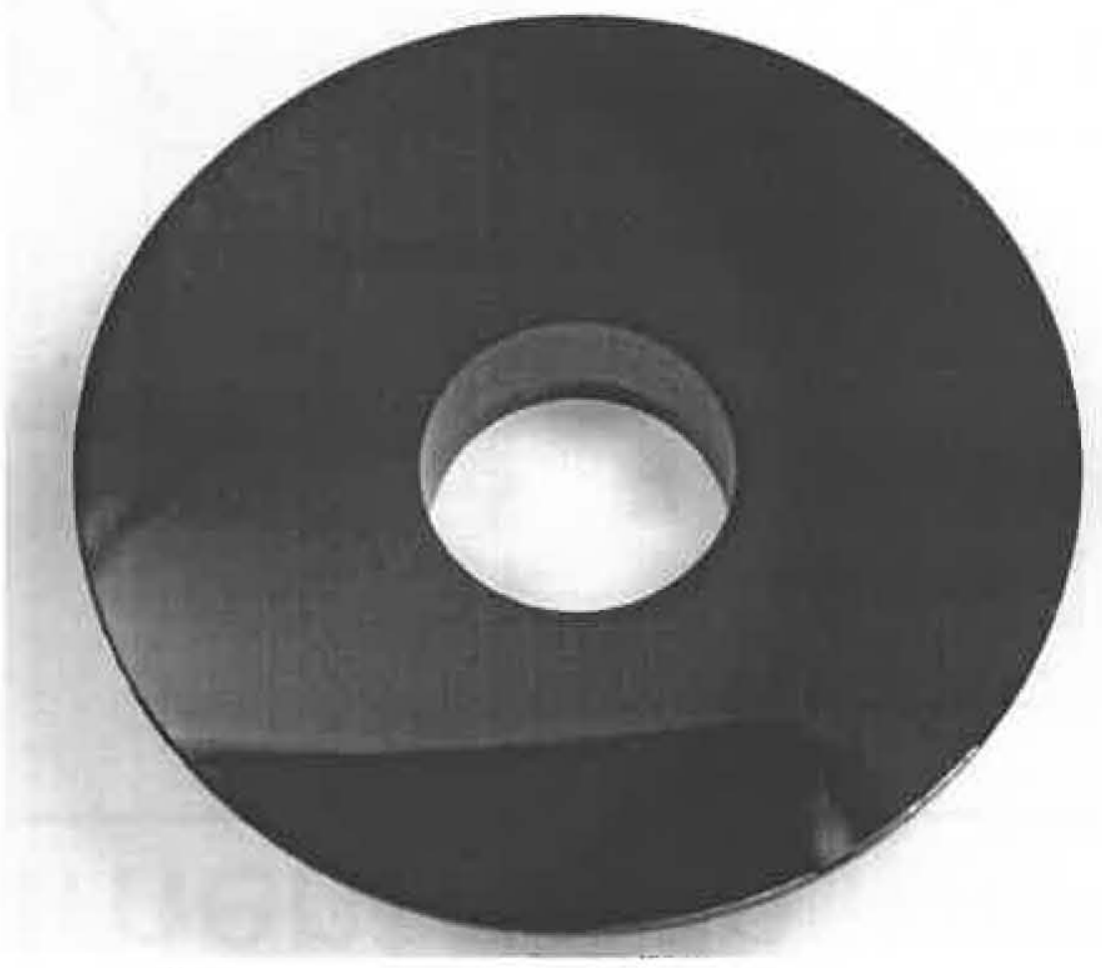

Front View (photo) 


\section{Light weight silicon mirrors for space instrumentation}

\section{TIRS Scene Mirror / Backside View}

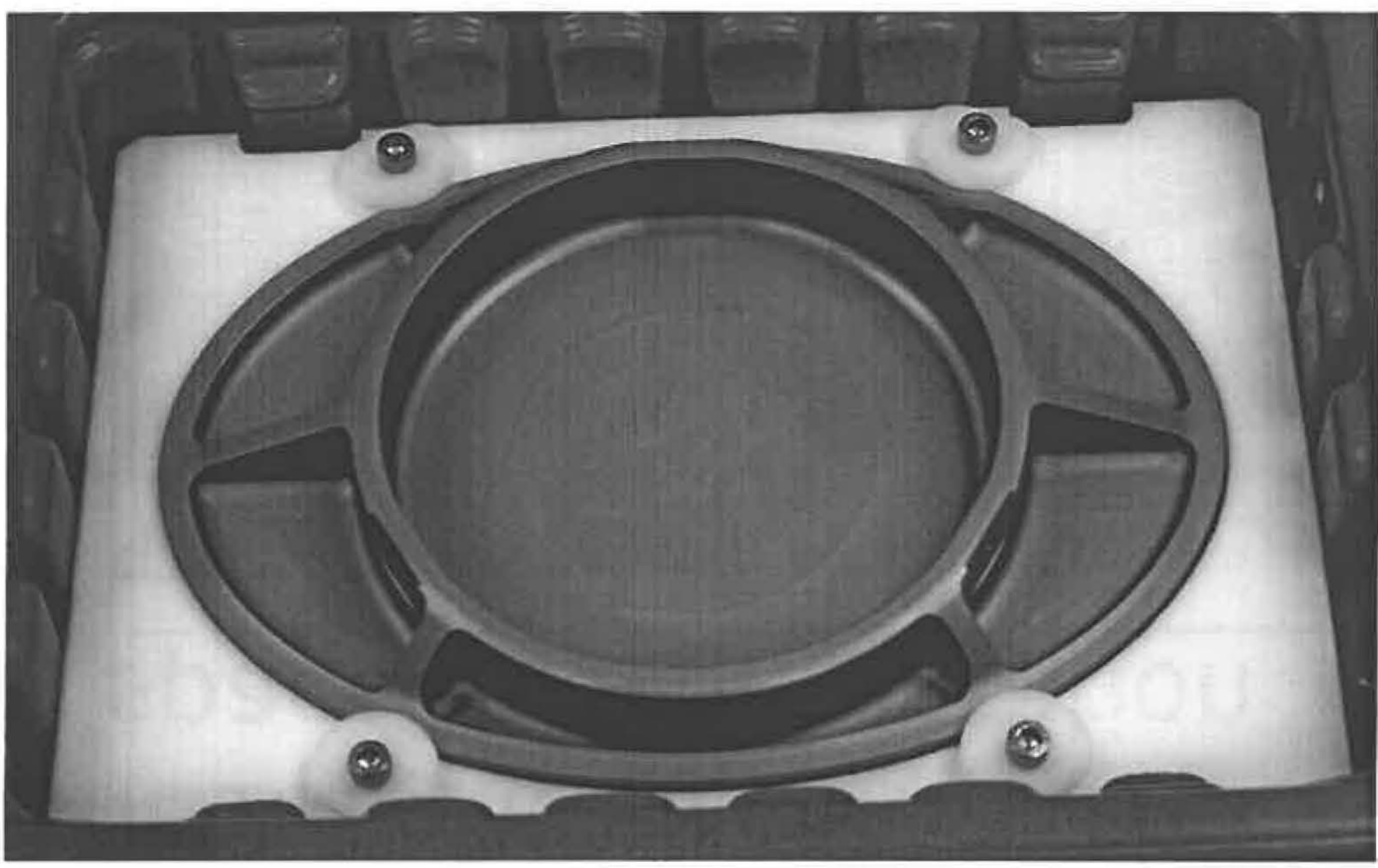




\section{Light weight silicon mirrors for space instrumentation}

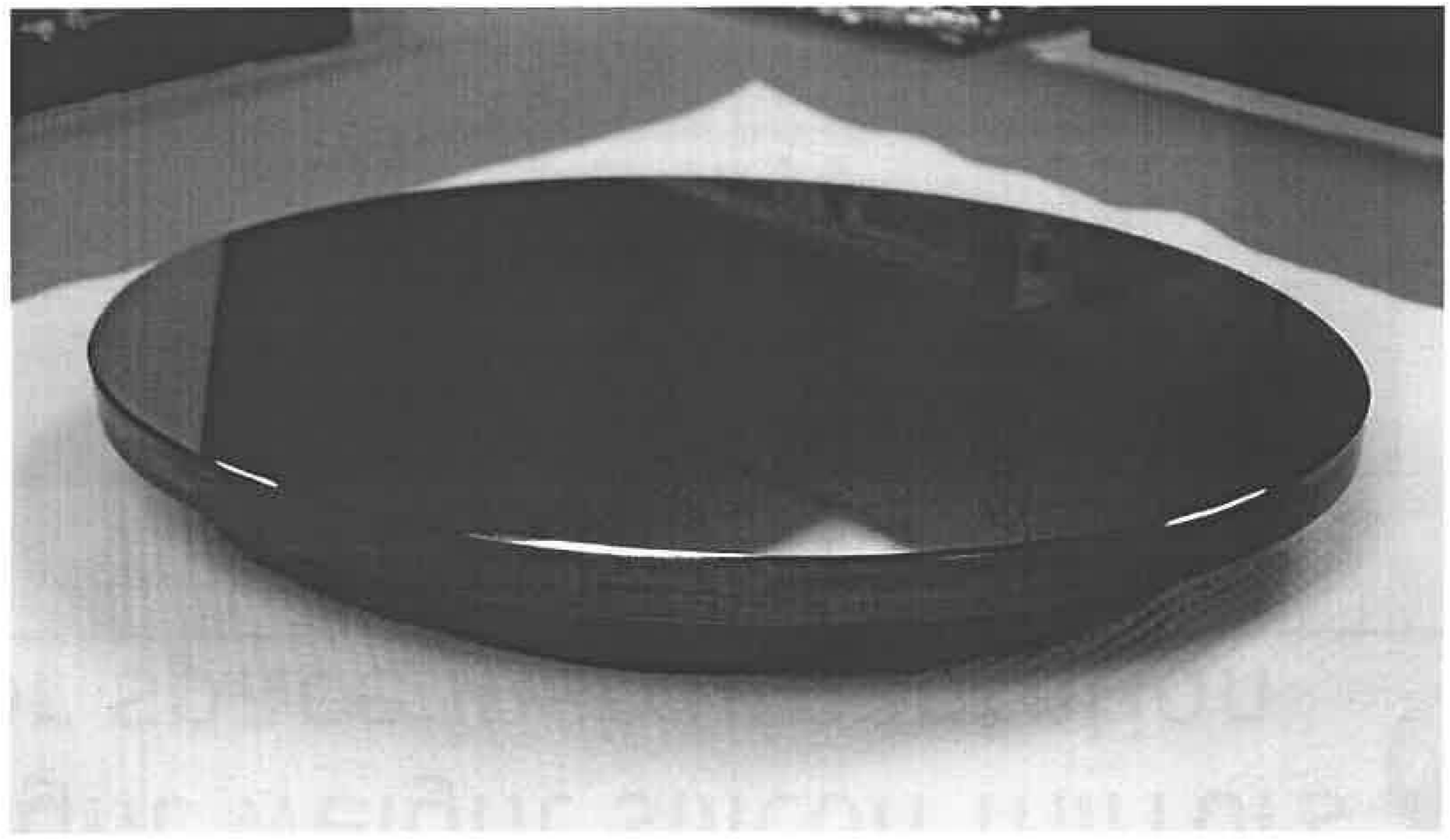




\section{Light weight silicon mirrors for space instrumentation}

\section{Cryogenic Test Facility}

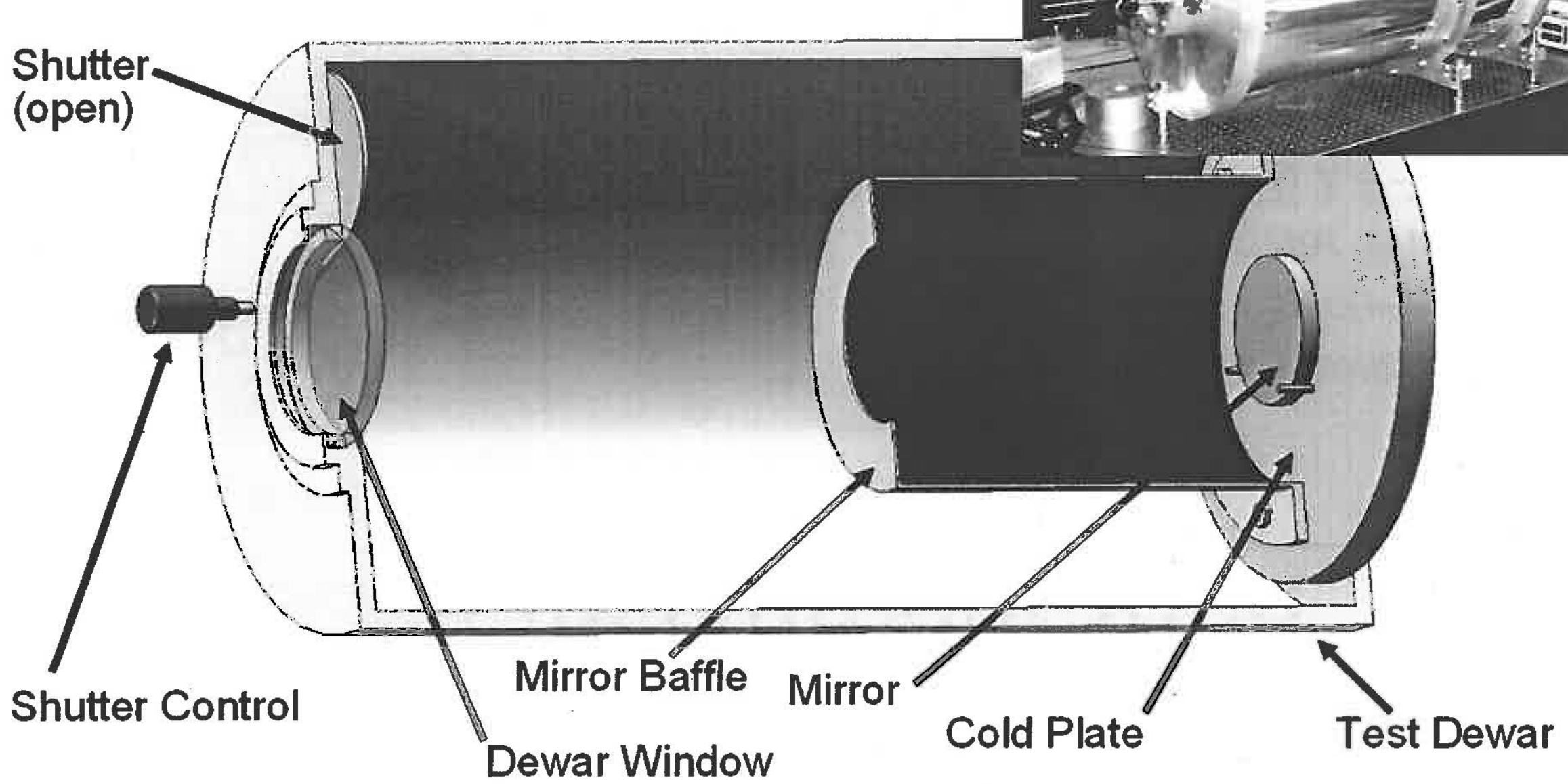




\section{Light weight silicon mirrors for space instrumentation}

Measured Change RT vs $83 \mathrm{~K}$ w/o Power $=0.001 \lambda$

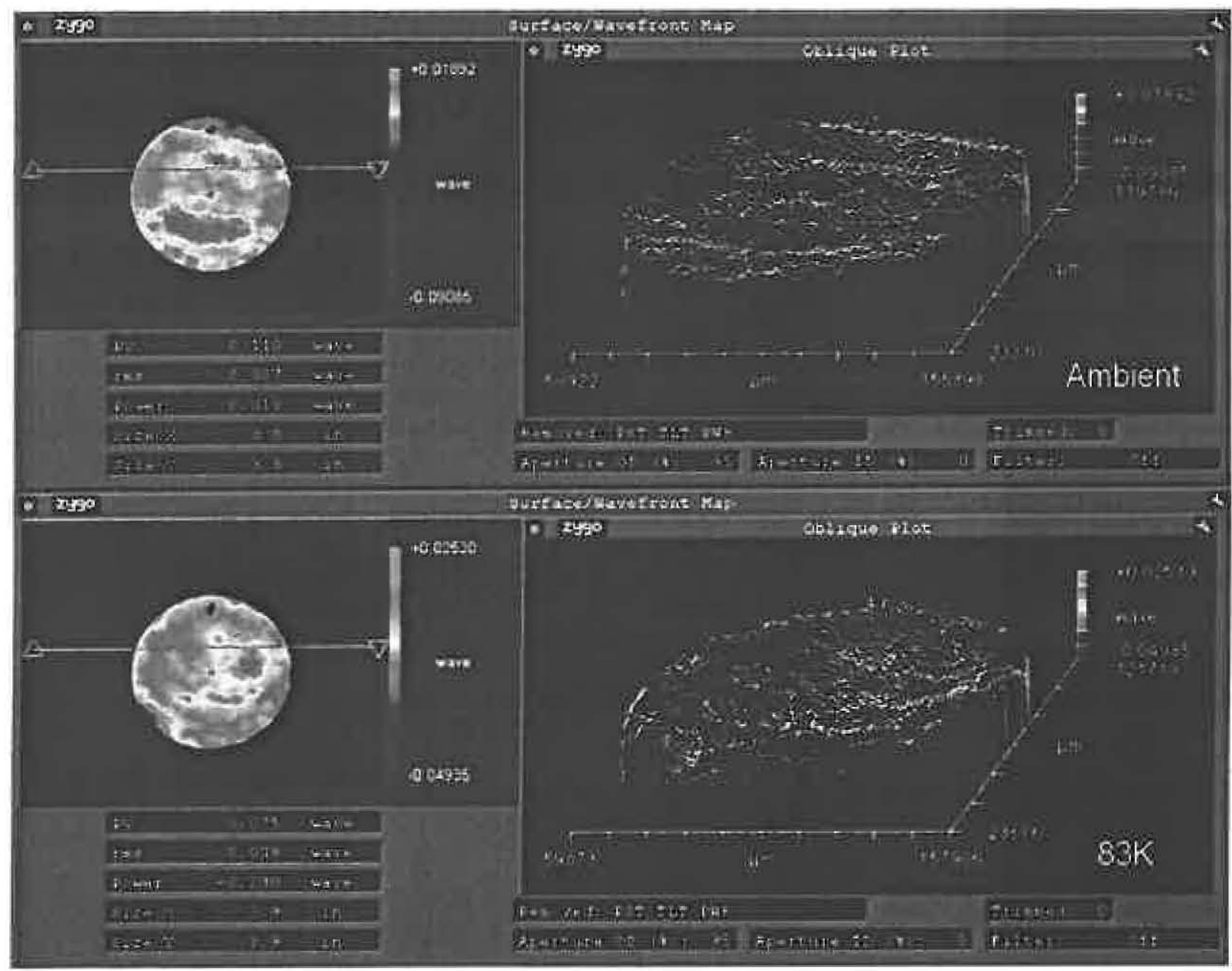

Actual Power Change After Compensating for Dewar Window $=0.056 \lambda \mathrm{P}-\mathrm{V}$, $=\lambda / 30 \mathrm{RMS}$. 


\section{Light weight silicon mirrors for space instrumentation}

Flat Mirror with Side Mounting Ferrules

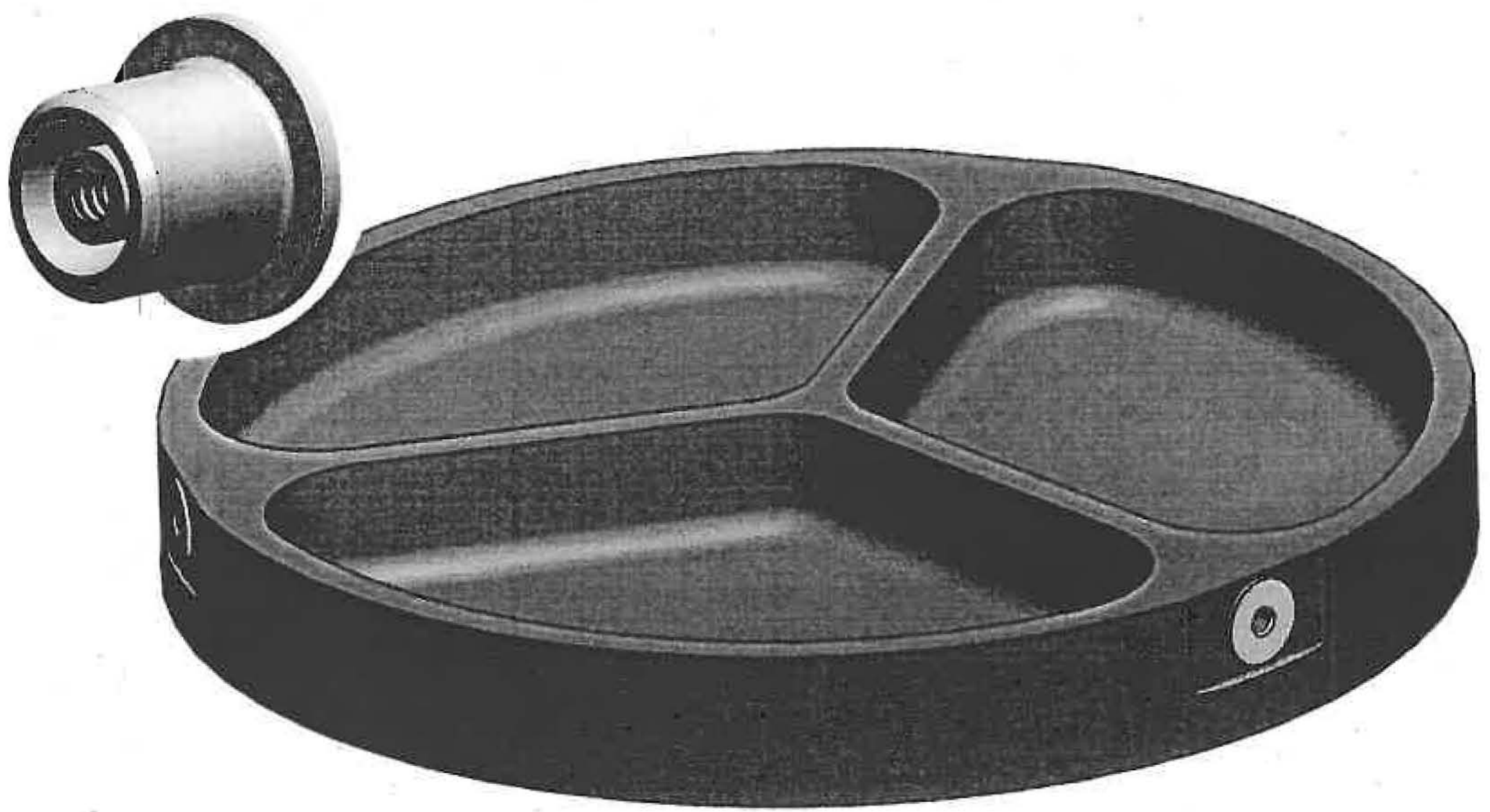


